\title{
Special Issue: Quantum Information and Quantum Logic
}

\author{
Junde Wu
}

Received: 2 March 2013 / Accepted: 4 March 2013 / Published online: 9 March 2013

(C) Springer Science+Business Media New York 2013

From the 2012 August 10 to 13, The International Symposium on Quantum Information and Quantum Logic was successfully held in Zhejiang University in Hangzhou of China. Professor Guilu Long, the famous expert in Quantum theory who came from Tsinghua University, served as the chairman of the workshop.

This is a high level symposium on Quantum theory. Many well known experts attended the meeting, among whom came from Tsinghua University, Chinese Academy of Sciences, University of Science and Technology of China, Beijing Normal University, Wuhan Institute of Mathematics and Physics of Chinese Academy of Sciences, Fudan University, Zhongshan University, Shandong University, Harbin Institute of Technology, Taiyuan University of Technology, Shanxi University, Shaanxi Normal University, Capital Normal University, Hebei Normal University, Beijing University of Posts and Telecommunications, China University of Petroleum, Harbin University of Science and Technology, China Jiliang University, Hangzhou Dianzi University, Yanshan University, Guilin University of Technology, Shenyang University of Technology, Yanbian University, Guangdong Education Institute, University of Salento, Technische University Darmstadt, Nagoya University, University of Bristol, National University of Singapore, Università degli Studi di Salerno and Zhejiang University.

60-minute special lectures and 30-minute achievement reports are the main forms of the conference. There are 19 special and 7 achievement reports in all. During the meeting, the Faculty of Science of Zhejiang University also invited the chairman of the symposium Professor Guilu Long and the Doctor Jochen Rau to give 60-minute outstanding scholar reports respectively.

The Guest Editor would like to express his personal gratitude to all of the organizers of the meeting and all the authors who contributed to the Special Issue.

This project is supported by Natural Science Foundation of China, 11210301022 and 11171301.

J. Wu (凶)

Department of Mathematics, Zhejiang University, Hangzhou 310027, People's Republic of China

e-mail:wjd@zju.edu.cn 


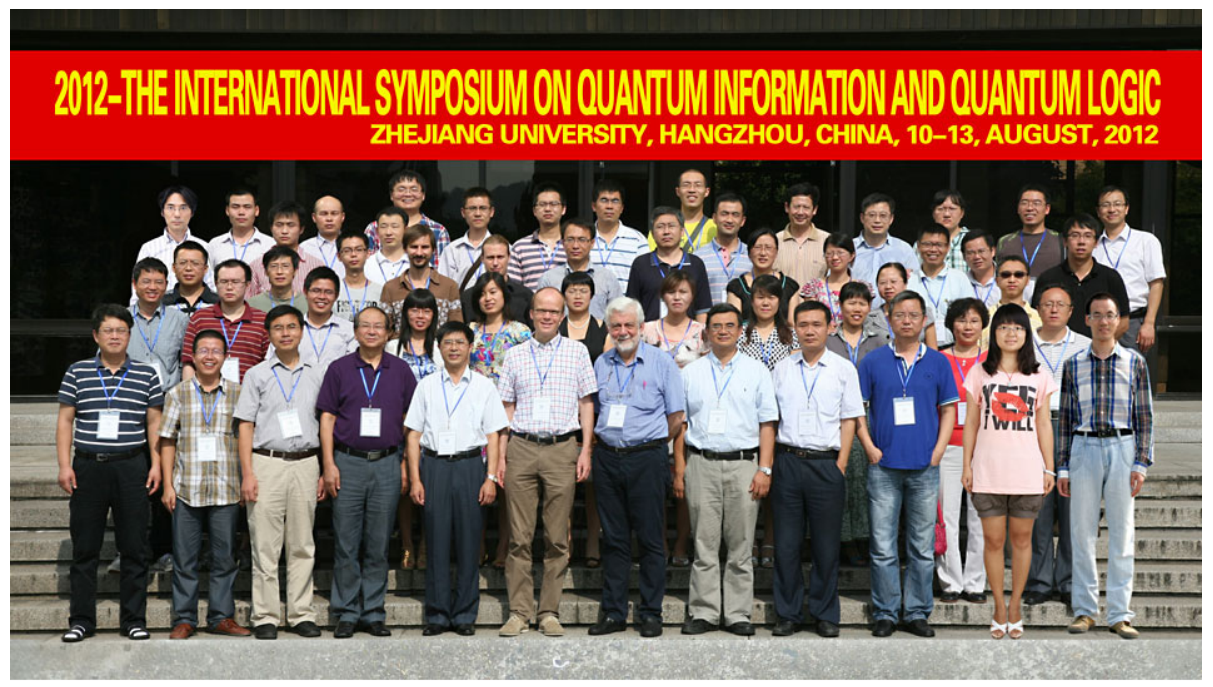

2012 Quantum conference in Zhejiang University, Hangzhou, China 